Methods: We conducted an analysis of three indirect indicators of quality of care: Need for reassessment due to pain (NRD), specialised referral rate (TDE) and length of stay in the emergency room (TDU). Records of patients treated for acute omalgia between 2014 and 2016 were reviewed and classified according to whether or not ultrasound was used as a diagnostic tool. For the statistical analysis we used the Student's T test, Fisher's exact test and survival curve (for TDU, TDE and NRD, respectively). The design used was that of a retrospective descriptive study. The information on waiting times was obtained from the CAJAL registry, information on new consults and specialised referral registration were obtained from the HORUS program and CAJAL registry. A comparative retrospective study was carried out based on the usual clinical practice. We examined the records of patients treated between 2015-2016 in the rheumatological emergency unit, who were known diabetics and who underwent an ultrasound-guided infiltration. Using the HORUS program we reviewed the patient's files of the immediate outcome after infiltration and the follow up consults.

Results: We included 1433 records of patient. Of these, 547 (38.1\%) were examined with ultrasound assistance (Group-ECO) from which 2 were performed by a radiologist and the rest by a trained physician (Rheumatologist or Family Physician). The rest of the assessments, $886(61.8 \%)$ were made without using the ultrasound (Control group), although in 540 cases a different imaging test was used. At 30 days after the first assessment, 90 patients $(10.1 \%)$ in the control group had consulted again (56 due to emergencies and 34 per AP), while in the ECO-Group $14(2.5 \%)$ consulted again at least once (12 for emergencies and 2 for AP) $(p<0.001)$. The referral rate to the specialist in the Control-Group was $36.5 \%$, while in the ECO-Group was $6.21 \%(p<0.0001)$. The average stay time was 94.5 DE 34.3 min in the Control Group and 105.4 DE 40.1 min in the ECOGroup $(p<0.0001)$

Conclusions: The use of ultrasound as a diagnostic tool reduces the need for reevaluations of patients and the rate of referrals to the specialist in a significant way. On the contrary, it increases the patient's stay time in the emergency room, probably due to delays in the availability of the equipment to perform the diagnostic procedure. Globally, our results show that most quality health care indexes improve in patients assessed by using ultrasonography.

Disclosure of Interest: None declared

DOI: 10.1136/annrheumdis-2018-eular.7215

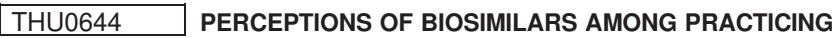 RHEUMATOLOGISTS: RESULTS FROM A MULTICOUNTRY STUDY IN EUROPE}

C. Karki ${ }^{1}$, S. Shah ${ }^{1}$, L. Archer ${ }^{2}$, A. Baskett ${ }^{2}$, E. Baynton ${ }^{2}$, L. Ha ${ }^{1}$, B. Shah-Manek ${ }^{3}$ ${ }^{1}$ Ipsos Healthcare, New York, USA; ${ }^{2}$ Ipsos Healthcare, London, UK; ${ }^{3}$ Ipsos Healthcare, San Francisco, USA

Background: In recent years, a substantial number of biosimilar (BioSm) products have come into the market for the treatment of rheumatic disorders, that might potentially improve patient access to treatment, resulting in cost savings and improved outcomes. However, given the limited regulatory evidence, clinical decision making continues to be a challenge.

Objectives: The objective of the study was to assess perceptions of prescribing biosimilars among rheumatologists and gain insights on barriers to access in the EU5 countries (United Kingdom (UK), France (FR), Italy (IT), Spain (SP) and Germany (DE)).

Methods: A cross-sectional survey was conducted in Q4 2016 in the EU5 among national, regional and hospital physicians who had been practicing between 3-30 years. Respondents completed a Physician Biosimilar Perceptual Questionnaire, which assessed the overall perception of anti-TNF biosimilars, specific barriers to prescribing them and cost factors influencing the current use and adoption of biosimilars. Data was analysed using descriptive statistics.

Results: A total of 256 rheumatologists in the EU5 were recruited as part of the study (with almost equal number of rheumatologists representing each EU5 country). Treating rheumatologists were practicing for an average of 16 years, mainly practicing in teaching hospitals (52\%), and urban hospitals (21.9\%). Overall, $83 \%$ of rheumatologists had awareness of biosimilars (range: $70 \%$ in DE and IT to $92 \%$ in UK). Among the different domains queried, only one in four rheumatologists trusted BioSm as much as branded biologics (range: $17.3 \%$ in IT to $39.2 \%$ in UK) and about $30 \%$ were comfortable with the safety profiles, and reported that long term efficacy and safety was of great importance for prescribing BioSm. Comparing perceptions of BioSm across countries, only $25 \%$ of rheumatologists in IT felt that BioSm offered equivalent efficacy to branded biologics, whereas $60.8 \%$ in the UK agreed with the same statement (figure 1). There was a consensus among respondents in all five countries regarding the lower cost of BioSm (range: $56.9 \%$ in SP to $72.5 \%$ in UK). Over $35 \%$ of the rheumatologists anticipated that clinical guidelines would require them to prescribe biosimilars. Data also showed that $63 \%$ chose BioSm for cost and $49 \%$ for formulary placement in clinical practice.

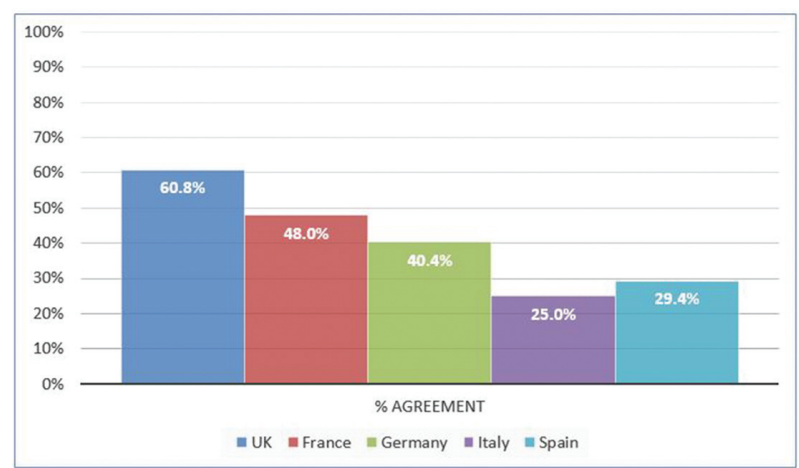

Abstract THU0644 - Figure 1 Perceptions. of Physicians in EUS: Equivalent efficacy as branded biologics

Conclusions: Overall, rheumatologists expressed the greatest level of awareness of biosimilars, even though differences were seen among the countries Long term safety and efficacy data was of key importance; however, cost and formulary coverage did impact the prescribing pattern.

Disclosure of Interest: C. Karki Employee of: Ipsos Healthcare, S. Shah Employee of: Ipsos Healthcare, L. Archer Employee of: Ipsos Healthcare, A. Baskett Shareholder of: ImmuPharma, Employee of: Ipsos Healthcare, E. Baynton Employee of: Ipsos Healthcare, L. Ha Employee of: Ipsos Healthcare, B. ShahManek Employee of: Ipsos Healthcare

DOI: 10.1136/annrheumdis-2018-eular.3751

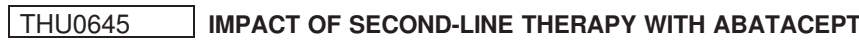 VERSUS OTHER TARGETED DMARDS ON THE RISK FOR INFECTION-RELATED HOSPITALIZATIONS AND ASSOCIATED COSTS AMONG RA PATIENTS IN THE UNITED STATES}

D. Paul ${ }^{1}$, L. McDonald ${ }^{1}$, A. Marshall ${ }^{1}$, T. Curtice ${ }^{1}$, M. Lingohr-Smith ${ }^{2}$, B. Menges ${ }^{2}$ J. Lin ${ }^{2} .{ }^{1}$ Bristol-Myers Squibb, Princeton; ${ }^{2}$ Novosys Health, Green Brook, USA

Background: Abatacept is a targeted DMARD (tDMARD) with a unique mechanism of action that has demonstrated a lower risk of infection-related hospitalizations as a second-line (2L) therapy compared with other tDMARDs among patients (pts) with RA. The cost savings in the United States associated with this reduced risk of infection-related hospitalizations is not well understood.

Objectives: To compare infection-related hospitalisation risk and associated healthcare costs of RA pts who were treated at $2 \mathrm{~L}$ with abatacept versus other tDMARDs, including TNF- $\alpha$ inhibitors (TNFis) and non-TNFis.

Methods: Pts prescribed a $2 \mathrm{~L}$ tDMARD (index date) who had $\geq 1$ inpatient diag nosis or $\geq 2$ outpatient diagnoses of RA in the 12 months prior to the index date were identified from the MarketScan ${ }^{\oplus}$ Commercial and Medicare claims databases between 1 January 2010 and 30 September 2015. Pts were required to have 12 months of continuous insurance coverage prior to the index date (baseline period) and throughout the follow-up period ( $\geq 12$ and up to 36 months). All pts were treated with a TNFi in first line. Infection-related hospitalizations and associated costs (on a per-pt per-month [PPPM] basis) were evaluated during baseline and follow-up periods. Multivariable regression analyses were used to evaluate the impact of index drugs on the risk of infection-related hospitalisation, as well as infection-related medical cost change from baseline to follow-up periods, while controlling for differences in baseline pt characteristics.

\begin{tabular}{|c|c|c|c|c|}
\hline \multicolumn{5}{|c|}{ Unadjusted Comparison of Medical Cost, PPPM } \\
\hline & Abatacept & TNFis & Non-TNFis & p-value \\
\hline Baseline medical costs & $\$ 121$ & $\$ 48$ & $\$ 39$ & 0.02 \\
\hline Follow-up medical costs & $\$ 30$ & $\$ 69$ & $\$ 102$ & 0.16 \\
\hline $\begin{array}{l}\text { Medical cost difference } \\
\text { (follow-up - baseline) }\end{array}$ & $-\$ 91$ & $\$ 21$ & $\$ 63$ & 0.005 \\
\hline \multicolumn{5}{|c|}{ Regression Adjusted Comparison of Difference of Medical Cost, PPPM } \\
\hline TNFi vs abatacept & \multicolumn{3}{|c|}{$\$ 51$} & 0.09 \\
\hline Non-TNFi vs abatacept & \multicolumn{3}{|c|}{$\$ 76$} & 0.04 \\
\hline
\end{tabular}

Results: Among the study population, 285 pts (mean age 55 years; $85 \%$ female) were treated with abatacept, 954 with TNFis (mean age 52 years; $82 \%$ female) and 288 with non-TNFis (mean age 55 years; $76 \%$ female). After controlling for differences in pts' characteristics, Cox regression showed that the risk for an infection-related hospitalisation was significantly greater among those treated with TNFis (hazard ratio $[\mathrm{HR}] 2.8 ; 95 \% \mathrm{Cl} 1.2,6.7 ; \mathrm{p}=0.02$ ) and higher, although not 
significant, among those treated with other non-TNFis (HR 2.4; 95\% Cl 0.9, 6.3; $\mathrm{p}=0.08$ ) compared with those treated with abatacept. Infection-related medical costs were lower for abatacept compared with other non-TNFis as shown in the table 1 .

Conclusions: In the real-world setting in the United States, pts treated with abatacept versus other tDMARDs tended to have a lower risk for infection-related hospitalisation and lower infection-related medical costs.

Disclosure of Interest: D. Paul Employee of: Bristol-Myers Squibb, L. McDonald Employee of: Bristol-Myers Squibb, A. Marshall Shareholder of: Bristol-Myers Squibb, Employee of: Bristol-Myers Squibb, T. Curtice Employee of: Bristol-Myers Squibb, M. Lingohr-Smith Grant/research support from: Bristol-Myers Squibb, Employee of: Novosys Health, B. Menges Grant/research support from: BristolMyers Squibb, Employee of: Novosys Health, J. Lin Grant/research support from: Bristol-Myers Squibb, Employee of: Novosys Health

DOI: 10.1136/annrheumdis-2018-eular.2323

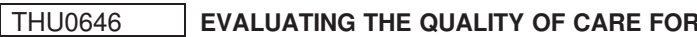 RHEUMATOID ARTHRITIS}

C. Barber ${ }^{1}$, D. Marshall ${ }^{1}$, E. Szefer ${ }^{2}$, D. Thompson ${ }^{2}$, D. Lacaille ${ }^{3} .{ }^{1}$ University of Calgary, Calgary; ${ }^{2}$ Emmes Canada; ${ }^{3}$ University of British Columbia, Vancouver, Canada

Background: The Arthritis Alliance of Canada (AAC) has developed performance measures (PMs) to evaluate RA care quality.

Objectives: To operationalize and report on 4 PMs using administrative data for British Columbia (BC), Canada: PM1) percentage of patients with new onset RA with at least one visit to a rheumatologist in the first year after diagnosis, PM2) percentage of RA patients under the care of a rheumatologist seen in follow-up at least once per year, PM3) percentage of RA patients dispensed a disease modifying anti-rheumatic drug (DMARD) during the measurement year, PM4) time to DMARD therapy in new onset RA.

Methods: All patients with RA visits between 01/01/1997 and 31/12/2009 in BC were identified using health administrative data and followed until December 2014. Cases were identified by $\geq 2$ physician billing codes for $R A \geq 8$ weeks but $\leq 5$ years apart. For this study, only cases age $>18$ who were seen by a rheumatologist at some point over follow-up were included. PM1: The percentage of incident RA cases with at least one visit to a rheumatologist within one year of their first RA visit was evaluated. PM2: The proportion of prevalent RA cases having at least one visit per year was calculated for those under rheumatology care. PM3: The percentage of prevalent RA patients dispensed a DMARD (including biologic agents and small molecule inhibitors) was calculated. PM4: time from RA onset (defined as first RA visit) to DMARD therapy was reported (in the calendar year of RA incidence), using median and 90th percentile wait time, as well as the proportion meeting the benchmark of 14 days.

Results: The cohort included 18976 incident and 29639 prevalent RA cases. The percentage of patients seen by a rheumatologist within 1 year from RA diagnosis increased over time, while the percentage of RA patients under the care of a rheumatologist seen in yearly follow-up declined steadily overtime. Further analysis (data not shown) suggests this is due to having more people with longer followup in the latter years, and lost to follow-up increasing over time, rather than a true calendar year effect. The percentage of RA patients dispensed a DMARD was suboptimal $(56 \%>65 \%)$. Of note, patients were not necessarily seen by a rheumatologist during the measurement year. The median time to DMARD improved over time to 23 days in 2009, with roughly one third receiving a DMARD within the benchmark of 14 days.

Conclusions: The present study represents the first time the AAC's PMs have been tested in administrative data and highlights where the measures are being met and potential gaps in care which require further examination.

Disclosure of Interest: None declared

DOI: 10.1136/annrheumdis-2018-eular.5023

\section{THU0647 LONGITUDINAL STUDY OF LONG-TERM POVERTY AND PERSISTENT DEPRESSIVE SYMPTOMS IN SLE}

E. Yelin ${ }^{1}$, J. Yazdany ${ }^{1}$, L. Trupin ${ }^{1}$, N. McCormick ${ }^{2}$, P. Katz ${ }^{1}$. 'Medicine/

Rheumatology, University of California, San Francisco, San Francisco, USA;

${ }^{2}$ Arthritis Research Centre of Canada, University of British Columbia, Vancouver, Canada

Background: A prior study found that persons with SLE in long-term poverty have greater accumulation of disease damage over 6 years than those exiting poverty or never in poverty ${ }^{1}$. The present study evaluates the effect of long-term poverty status on depressive symptoms over the same duration of time.

Objectives: Analyse the impact of long-term poverty on prevalent and incident persistent depression after accounting for other risk factors for depression among persons with SLE.

Methods: Data are from the UCSF Lupus Outcomes Study in which persons with SLE were recruited in 2003 throughout the U.S. and interviewed annually through 2015. In each year we characterised respondents' poverty status based on household income and family size and administered the CESD measure of depressive symptoms, defining a high level of depressive symptoms using a validated SLEspecific cutpoint $(\geq 24)$ associated with a formal diagnosis of depression ${ }^{2}$. Prevalent persistent depression was defined as having high levels of depressive symp toms for $\geq 3$ years between 2009 and 2015. Incident persistent depression used the same criteria, measured only among those who had low levels of depressive symptoms between 2006 and 2009. Logistic regression was used to estimate the impact of being poor in every year from 2003-2009, permanently leaving poverty by 2009 , or never being poor on prevalent and incident persistent depression, with and without adjustment for gender, age, marital status, race/ethnicity, education, disease duration, extent of accumulated damage by 2009 using the Brief Index of Lupus Damage ${ }^{3}$, smoking status, and BMI.

Results: 535 persons with SLE were interviewed in each year from 2003 to 2015 (94\% female, 65\% non-Hispanic whites, mean age in 200350 years, range 20 83 , mean disease duration 17 years, range 1-51). Between 2003 and 2009, 81\% were never poor, $8 \%$ exited poverty, and $11 \%$ were poor in every year. 89 of the $535(16.6 \%)$ met the study definition of prevalent persistent depression; $23(7.4 \%)$ of the 312 free of high levels of depressive symptoms from 2006-2009 had incident persistent depression as of 2015. Table 1, below, indicates that those who were poor in every year had significantly higher rates of prevalent and incident persistent depression than those exiting poverty or never poor.

Abstract THU0646 - Table 1. Results of Four Performance Measures for RA.

\begin{tabular}{|c|c|c|c|c|c|c|c|}
\hline $\begin{array}{l}\text { Measurement } \\
\text { Year }^{1}\end{array}$ & $\begin{array}{l}\text { Prevalent } \\
\text { RA cases }\end{array}$ & $\begin{array}{l}\text { Incident } \\
\text { RA cases }\end{array}$ & $\begin{array}{l}\text { PM1: } \\
\text { Patients seeing rheumatologist } \\
\text { within } 1 \text { year of RA onset }\end{array}$ & $\begin{array}{l}\text { PM2:\% of Patients under rheum care } \\
\text { with yearly rheumatology visits }\end{array}$ & $\begin{array}{c}\text { PM3: } \\
\% \text { Patients } \\
\text { dispensed a } \\
\text { DMARD }\end{array}$ & $\begin{array}{c}\text { PM4: } \\
\text { Median } \\
\text { (90th percentile) } \\
\text { days to DMARD }\end{array}$ & $\begin{array}{c}\text { PM4: } \\
\% \text { with } \\
\text { DMARD } \\
\text { within } \\
14 \text { days of } \\
\text { onset }\end{array}$ \\
\hline 2004 & 17472 & 1647 & $80 \%$ & $57 \%$ & $59 \%$ & $31(825)$ & $27 \%$ \\
\hline 2005 & 19097 & 1614 & $82 \%$ & $56 \%$ & $60 \%$ & $26(579)$ & $30 \%$ \\
\hline 2006 & 20612 & 1704 & $85 \%$ & $54 \%$ & $60 \%$ & $23(411)$ & $31 \%$ \\
\hline 2007 & 21764 & 1430 & $83 \%$ & $53 \%$ & $60 \%$ & 29 (399) & $28 \%$ \\
\hline 2008 & 22721 & 1305 & $86 \%$ & $50 \%$ & $60 \%$ & 26 (339) & $29 \%$ \\
\hline 2009 & 23386 & 921 & $92 \%$ & $49 \%$ & $61 \%$ & $23(188)$ & $34 \%$ \\
\hline 2010 & 23122 & - & - & $46 \%$ & $60 \%$ & - & - \\
\hline 2011 & 22781 & - & - & $44 \%$ & $56 \%$ & - & - \\
\hline 2012 & 22370 & - & - & $42 \%$ & $56 \%$ & - & - \\
\hline 2013 & 21966 & - & - & $41 \%$ & $57 \%$ & - & - \\
\hline 2014 & 21412 & - & - & $41 \%$ & $57 \%$ & - & - \\
\hline
\end{tabular}

${ }^{1}$ PMs reported on a prevalent cohort until 2014, and on an incident cohort reported until 2009 